\title{
Numerical Aspects of Uniform Airy-type Asymptotic Expansions
}

\section{N. M. TEMME}

\begin{abstract}
An algorithm for evaluating Airy-type asymptotic representations of a class of special functions is discussed. The method is quite general, but the paper concentrates on Bessel functions. The algorithm is based on the differential equation of the Bessel functions, which has a turning point when order and argument of the Bessel functions are equal.
\end{abstract}

\section{INTRODUCTION}

From a computational point of view Airy-type uniform asymptotic expansions are unattractive. This is mainly due to the complexity of the coefficients in the expansions. In all known cases, for example Bessel functions, the coefficients are difficult to compute in the neighborhood of the turning point. Although in that case there are closed expressions for the coefficients in terms of elementary functions, there is massive cancellation in evaluating these expressions near the turning point.

In this paper we discuss a new numerical algorithm for uniform asymptotic representations of a class of functions. The method is quite general, but we only treat the case of Bessel functions. We use differential equations for functions representing (in an exact sense) the asymptotic series, and we base a numerical algorithm directly on these differential equations. Extra features are that we deal with convergent expansions, that we need only a small number of precomputed tabulated numbers, and that the method is applicable for quite small values of the large parameter. In some sense the method is similar to the one described for the computation of incomplete gamma functions in [4]. In that case the error function is the main approximant.

\section{Airy-type asymptotics of ORDINARY BESSEL FUnCtions}

The ordinary Bessel functions $J_{\nu}(z)$ and $Y_{\nu}(z)$ can be expanded in terms of Airy functions. From [3, p. 425], we obtain

$$
\begin{aligned}
& J_{\nu}(\nu z) \sim \frac{\phi(\zeta)}{\nu^{1 / 3}}\left[\operatorname{Ai}\left(\nu^{2 / 3} \zeta\right) \sum_{s=0}^{\infty} \frac{A_{s}(\zeta)}{\nu^{2 s}}+\nu^{-4 / 3} \operatorname{Ai}^{\prime}\left(\nu^{2 / 3} \zeta\right) \sum_{s=0}^{\infty} \frac{B_{s}(\zeta)}{\nu^{2 s}}\right], \\
& Y_{\nu}(\nu z) \sim-\frac{\phi(\zeta)}{\nu^{1 / 3}}\left[\operatorname{Bi}\left(\nu^{2 / 3} \zeta\right) \sum_{s=0}^{\infty} \frac{A_{s}(\zeta)}{\nu^{2 s}}+\nu^{-4 / 3} \operatorname{Bi}^{\prime}\left(\nu^{2 / 3} \zeta\right) \sum_{s=0}^{\infty} \frac{B_{s}(\zeta)}{\nu^{2 s}}\right],
\end{aligned}
$$

as $\nu \rightarrow \infty$, uniformly with respect to $z \in[0, \infty)$. The expansions are valid for complex values of $\nu$ and $z$, but here we concentrate on real values of the parameters.

1991 Mathematics Subject Classification. Primary 65D20; Secondary 41A60, 33C10, 34E20.

Key words and phrases. Uniform asymptotic expansions, computation of special functions, turning points, Airy-type expansions, Bessel functions.

The final version of this paper will be submitted for publication elsewhere. 
The parameter $\zeta$ is defined by

$$
\begin{aligned}
\frac{2}{3} \zeta^{3 / 2} & =\ln \frac{1+\sqrt{1-z^{2}}}{z}-\sqrt{1-z^{2}}, \quad 0 \leq z \leq 1, \\
\frac{2}{3}(-\zeta)^{3 / 2} & =\sqrt{z^{2}-1}-\arccos \frac{1}{z}, \quad z \geq 1 .
\end{aligned}
$$

Furthermore,

$$
\phi(\zeta)=\left(\frac{4 \zeta}{1-z^{2}}\right)^{1 / 4}
$$

The coefficients $A_{s}, B_{s}$ are given in [1, p. 368], and [3, p. 425], with

$$
A_{0}(\zeta)=1, \quad B_{0}(\zeta)=-\frac{5}{48 \zeta^{2}}+\frac{\phi^{2}(\zeta)}{2 \zeta}\left[\frac{5}{24\left(1-z^{2}\right)}-\frac{1}{8}\right]
$$

The coefficients $A_{s}, B_{s}$ in (2.1) are complicated expressions. In particular, they are regular but rather difficult to compute near the turning point $z=1$, or equivalently, near $\zeta=0$. In [2] all needed coefficients $A_{s}, B_{s}$ are expanded in Maclaurin series at the turning point, the power series being in terms of the variable $w^{2}=1-z^{2}$. We propose a different approach, in which the direct computation of $A_{s}, B_{s}$ can be circumvented. We define two functions $F_{\nu}, G_{\nu}$ which represent the asymptotic series in the above representations of the Bessel functions:

$$
\begin{aligned}
& J_{\nu}(\nu z)=\phi(\zeta) \nu^{-1 / 3}\left[\operatorname{Ai}\left(\nu^{2 / 3} \zeta\right) F_{\nu}(\zeta)+\nu^{-4 / 3} \operatorname{Ai}^{\prime}\left(\nu^{2 / 3} \zeta\right) G_{\nu}(\zeta)\right] \\
& Y_{\nu}(\nu z)=-\phi(\zeta) \nu^{-1 / 3}\left[\operatorname{Bi}\left(\nu^{2 / 3} \zeta\right) F_{\nu}(\zeta)+\nu^{-4 / 3} \operatorname{Bi}^{\prime}\left(\nu^{2 / 3} \zeta\right) G_{\nu}(\zeta)\right]
\end{aligned}
$$

Olver's approach for deriving Airy-type expansions for the Bessel functions is based on the differential equation

$$
\frac{d^{2} W}{d \zeta^{2}}=\left[\nu^{2} \zeta+\psi(\zeta)\right] W
$$

where

$$
\psi(\zeta)=\frac{5}{16 \zeta^{2}}+\frac{\zeta z^{2}\left(z^{2}+4\right)}{4\left(z^{2}-1\right)^{3}}
$$

the relation between $\zeta$ and $z$ being given in (2.2). The quantities within the square brackets in (2.4) are two solutions of equation (2.5).

By using equation (2.5), we can derive the following system of differential equations for the functions $F_{\nu}(\zeta), G_{\nu}(\zeta)$ :

$$
\begin{aligned}
F^{\prime \prime}+2 \zeta G^{\prime}+G-\psi(\zeta) F & =0, \\
G^{\prime \prime}+2 \nu^{2} F^{\prime} & -\psi(\zeta) G=0,
\end{aligned}
$$

where primes denote differentiation with respect to $\zeta$. A Wronskian for the system (2.7) follows by eliminating the terms $\psi(\zeta) F_{\nu}(z)$ and $\psi(\zeta) G_{\nu}(z)$. This gives

$$
F^{\prime} G-F G^{\prime}+\zeta G^{2}-\nu^{2} F^{2}=-\nu^{2} .
$$

The constant on the right-hand side follows by taking $\zeta=0$ and by using the known behavior of the Bessel functions at the turning point; see [1, pp. 368 and 446]. 


\section{Evaluation of the Functions $F_{\nu}(\zeta), G_{\nu}(\zeta)$}

We solve the system of differential equations (2.7) by using analytical techniques. We expand the functions $F_{\nu}(\zeta), G_{\nu}(\zeta)$ in Maclaurin series. The singular points of the function $\psi(\zeta)$, and hence those of the differential equations, occur at

$$
\zeta^{ \pm}=\left(\frac{3}{2} \pi\right)^{2 / 3} e^{ \pm i \pi / 3}
$$

(see $\left[3\right.$, p. 421]). It follows that the radius of convergence of the series of $F_{\nu}(\zeta)$ and $G_{\nu}(\zeta)$ in powers of $\zeta$ equals $2.81 \ldots$

We expand

$$
F_{\nu}(\zeta)=\sum_{n=0}^{\infty} f_{n}(\nu) \zeta^{n}, \quad G_{\nu}(\zeta)=\sum_{n=0}^{\infty} g_{n}(\nu) \zeta^{n}, \quad \psi(\zeta)=\sum_{n=0}^{\infty} \psi_{n} \zeta^{n}
$$

The coefficients $f_{0}, f_{1}, \ldots, g_{0}, g_{1}, \ldots$ are to be determined, while the coefficients $\psi_{n}$ are known. The first few $\psi_{n}$ are

$$
\psi_{0}=\frac{1}{70} 2^{1 / 3}, \quad \psi_{1}=\frac{2}{75}, \quad \psi_{2}=\frac{69}{13475} 2^{2 / 3}, \quad \psi_{3}=-\frac{148}{73125} 2^{1 / 3} .
$$

Upon substituting the expansions into (2.7), we obtain for $n=0,1,2, \ldots$ the system of recursion relations

$$
\begin{array}{ll}
(n+2)(n+1) f_{n+2}+(2 n+1) g_{n}=\phi_{n}, & \phi_{n}=\sum_{k=0}^{n} f_{k} \psi_{n-k}, \\
(n+2)(n+1) g_{n+2}+2 \nu^{2}(n+1) f_{n+1}=\chi_{n}, & \chi_{n}=\sum_{k=0}^{n} g_{k} \psi_{n-k} .
\end{array}
$$

The system of recursions in (3.3) is not stable in forward direction (especially when $\nu$ is large). When we try to use (3.3) in backward direction, for instance with false starting values $f_{N}, g_{N}$ for some large integer $N$, a complication arises because of the terms $\phi_{n}, \chi_{n}$ on the right-hand sides of (3.3). All terms $\phi_{n}, \chi_{n}$ contain $f_{k}, g_{k}$ for $k=0,1,2, \ldots, n$. Hence, recursion in backward direction is not possible at all. A way out is to consider $\phi_{n}, \chi_{n}$ as known quantities, and to treat (3.3) as a system of inhomogeneous difference equations.

A first step in this approach will be to solve the system (2.7) by iteration. That is, we choose an appropriate pair of functions $F_{0}, G_{0}$, and define two sequences of functions $\left\{F_{m}\right\},\left\{G_{m}\right\}$ by writing for $m=1,2,3, \ldots$ :

$$
\begin{aligned}
F_{m}^{\prime \prime}+2 \zeta G_{m}^{\prime}+G_{m} & =\psi(\zeta) F_{m-1}, \\
G_{m}^{\prime \prime}+2 \nu^{2} F_{m}^{\prime} & =\psi(\zeta) G_{m-1} .
\end{aligned}
$$

We rewrite (3.3) in backward form:

$$
\begin{aligned}
f_{n} & =\frac{1}{2 \nu^{2}}\left[\frac{1}{n} \chi_{n-1}-(n+1) g_{n+1}\right], \\
g_{n-1} & =\frac{1}{2 n-1}\left[\phi_{n-1}-n(n+1) f_{n+1}\right],
\end{aligned}
$$

where $n \geq 1$. The coefficients are assumed to belong to the functions $F_{m}(\zeta), G_{m}(\zeta)$ of the iteration process described by (3.4), while the coefficients $\phi_{n-1}, \chi_{n-1}$ are 
assumed to be known, and contain Maclaurin coefficients of $F_{m-1}(\zeta), G_{m-1}(\zeta)$ and $\psi(\zeta)$. Observe that (3.5) does not define $f_{0}$. After having computed

$$
f_{1}, f_{2}, \ldots, g_{0}, g_{1}, g_{2}, \ldots
$$

by the backward recursion process, we compute $f_{0}$ from the Wronskian (2.8):

$$
f_{0}=\frac{-g_{1}+\sqrt{g_{1}^{2}+4 \nu^{2}\left(\nu^{2}+f_{1} g_{0}\right)}}{2 \nu^{2}} \text {. }
$$

\begin{tabular}{|c|c|c|c|c|c|c|}
\hline \multicolumn{7}{|c|}{$\nu=25$} \\
\hline$i$ & $\left|f_{0}-f_{0}^{a}\right|$ & $\left|g_{0}-g_{0}^{a}\right|$ & $\left|f_{5}-f_{5}^{a}\right|$ & $\left|g_{5}-g_{5}^{a}\right|$ & $\left|f_{10}-f_{10}^{a}\right|$ & $\left|g_{10}-g_{10}^{a}\right|$ \\
\hline & & & & & & \\
\hline 1 & $1.12 e-11$ & $5.29 e-11$ & $4.33 e-12$ & $1.55 e-09$ & $1.45 e-13$ & $4.09 e-11$ \\
\hline 2 & $3.66 e-16$ & $4.00 e-13$ & $2.85 e-14$ & $8.86 e-14$ & $1.18 e-15$ & $5.32 e-15$ \\
\hline 3 & $0.00 e-00$ & $2.52 e-17$ & $2.66 e-18$ & $1.55 e-16$ & $1.25 e-18$ & $4.63 e-16$ \\
\hline 4 & $0.00 e-00$ & $0.00 e-00$ & $5.01 e-20$ & $1.46 e-19$ & $1.14 e-18$ & $4.48 e-16$ \\
\hline 5 & $0.00 e-00$ & $1.69 e-21$ & $5.53 e-20$ & $1.47 e-19$ & $1.14 e-18$ & $4.48 e-16$ \\
\hline
\end{tabular}

Table 1. Absolute errors during five iterations $(i)$ of $f_{0}, g_{0}, f_{5}, g_{5}, f_{10}, g_{10}$ compared with more accurate values $f_{0}^{a}$, etc.

\section{NUMERICAL EXPERIMENTS}

We have computed successive iterates of Maclaurin coefficients $f_{n}, g_{n}$ defined in (3.1) for different values of $\nu$. We have used simple starting values of the first set $\left\{f_{n}, g_{n}\right\}$, using the known behavior of the Maclaurin coefficients. During each iteration we start the backward recursions with $f_{n}=g_{n-1}=0, n \geq 16$, and we compute $f_{15}, g_{14}, f_{14}, g_{13}, \ldots$ by using (3.5). We recompute the coefficients $\phi_{k}, \chi_{k}, k=0,1,2, \ldots, 14$, using (3.3) with values $f_{k}, g_{k}$ obtained in the previous iteration. For $\nu=25$, Table 1 gives the errors in the values $f_{0}, g_{0}, f_{5}, g_{5}, f_{10}, g_{10}$, when compared with more accurate values $f_{0}^{a}$, etc.

From the table we conclude that for $\nu=25$ we can already obtain an accuracy of $10^{-13}$ after two iterations. The algorithm is very efficient, especially when $\nu$ becomes larger.

\section{REFERENCES}

1. M. Abramowitz and I.A. Stegun, Handbook of mathematical functions, Washington D.C., 1964.

2. D. Amos, Algorithm 644, A portable package for Bessel functions of a complex argument and nonnegative order, ACM Trans. Math. Software 12 (1986), 265-273.

3. F.W.J. Olver, Asymptotics and Special Functions, Academic Press, New York, 1974.

4. N.M. Temme, On the computation of the incomplete gamma functions for large values of the parameters, in Algorithms for approximation, Shrivenham, July 1985 (J. C. Mason and M.G. Cox, eds.), Clarendon, Oxford, 1987, pp. 479-489.

5. F.G. Tricomi, Repertorium der Theorie der Differentialgleichungen, Springer, Berlin, 1968.

CWI, P.O. Box 94079, 1090 GB Amsterdam, The Netherlands

E-mail address: nicot@cwi.nl 